\title{
Minimally invasive tricuspid valve surgery in patients at high risk
}

\author{
Davide Ricci, MD, ${ }^{\mathrm{a}, \mathrm{b}}$ Massimo Boffini, MD, ${ }^{\mathrm{a}}$ Cristina Barbero, MD, ${ }^{\mathrm{a}}$ Suad El Qarra, MD, ${ }^{\mathrm{a}}$ \\ Giovanni Marchetto, MD, ${ }^{a}$ and Mauro Rinaldi, $\mathrm{MD}^{\mathrm{a}}$
}

Objective: Reports of minimally invasive tricuspid valve operations are rare, and results are often contradictory. This study analyzes our 5-year experience with minimally invasive tricuspid valve operations in high-risk patients.

\begin{abstract}
Methods: Between November 2005 and December 2011, tricuspid valve surgery using a nonsternotomy minimally invasive technique was performed in 64 patients (19 male, 45 female; mean age, $63.2 \pm 12.8$ years). Mean preoperative European System for Cardiac Operative Risk Evaluation was $7.3 \pm 2.9$, and predicted mortality was $11.6 \% \pm 11.7 \%$. Tricuspid valve regurgitation cause was functional in 36 patients $(56.2 \%)$, endocarditis in 2 patients $(3.1 \%)$, and rheumatic in 24 patients $(37.5 \%)$. Two patients $(3.1 \%)$ showed prosthesis dysfunction. Forty patients $(62.5 \%)$ had undergone previous cardiac surgery.
\end{abstract}

Results: Tricuspid valve repair was performed in 35 patients (54.7\%). Tricuspid valve replacement with bioprosthesis was performed in 27 patients $(42.2 \%)$, and the remaining 2 patients $(3.1 \%)$ underwent bioprosthetic replacement. Concomitant procedures (48) included mitral valve surgery (42 patients), atrial septal defect closure (5 patients), and myxoma exeresis (1 patient). Conversion to sternotomy occurred in 1 patient $(1.6 \%)$. Overall hospital mortality was $7.9 \%$. Stroke occurred in 1 patient $(1.6 \%)$, and 5 patients underwent reoperation for bleeding (7.8\%). Mean follow-up time was $21 \pm 16$ months (range, 1-59 months) and 100\% completed. Cumulative Kaplan-Meier estimated 5-year survival was $81.3 \%$, and 5-year freedom from reoperation was $100 \%$.

Conclusions: The heart-port-based minimally invasive approach seems to be safe, feasible, and reproducible in case of tricuspid valve operations. It ensures low perioperative morbidity, moderate to low rates of tricuspid regurgitation recurrence, and low late mortality. It also seems to have an added value in case of reoperative procedures. (J Thorac Cardiovasc Surg 2014;147:996-1001)

Right-sided cardiac valvular disease has traditionally been considered less clinically important than mitral or aortic valve pathology, and its optimal management remains controversial. Patients are rarely referred for isolated surgical tricuspid valve (TV) repair or replacement, and most procedures are done in the context of other planned cardiac surgery.

However, significant tricuspid regurgitation (TR) can lead to functional impairment and has an adverse impact on perioperative outcomes, functional class, and survival. ${ }^{1}$ Approximately $90 \%$ of cases of TR are secondary to left heart pathology with various degrees of pulmonary hypertension, and approximately $10 \%$ are due to primary $\mathrm{TV}$

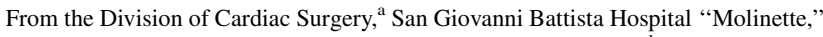
University of Turin, Turin, Italy; and Department of Surgery, ${ }^{\mathrm{b}}$ Dipartimento di Scienze Chirurgiche, Rianimatorie-Riabilitative e dei Trapianti d'Organo, Fondazione IRCCS “San Matteo” Hospital, University of Pavia, Pavia, Italy. Disclosures: Dr Rinaldi reports lecture fees from Novartis and Edwards Lifesciences. All other authors have nothing to disclose with regard to commercial support.

Received for publication Dec 14, 2012; revisions received March 4, 2013; accepted for publication March 6, 2013; available ahead of print Abril 29, 2013.

Address for reprints: Davide Ricci, MD, Division of Cardiac Surgery, San Giovanni Battista Hospital “Molinette," University of Torino, 10126 Torino, Italy (E-mail: daveri@libero.it).

0022-5223/\$36.00

Copyright (C) 2014 by The American Association for Thoracic Surgery http://dx.doi.org/10.1016/j.jtcvs.2013.03.018
}

disease. ${ }^{2}$ TR is generally well tolerated; however, in the presence of pulmonary hypertension, cardiac output declines and right heart failure worsens.

Although TR leads to a dismal prognosis after symptom development and in-hospital mortality and actuarial survival are improved in patients undergoing TV annuloplasty at the time of mitral valve (MV) surgery, TR remains frequently undertreated.

Because TR can vary according to the preload, afterload, and right ventricular function, ${ }^{3-5}$ the assessments of leaflet morphology, annular dimension (from the middle part of the septal annulus to the middle part of the anterior annulus), and pulmonary artery pressure are particularly important for determining subsequent management. It has been suggested that a tricuspid annulus diameter greater than $40 \mathrm{~mm}$ or $21 \mathrm{~mm} / \mathrm{m}^{2}$ measured in the 4-chamber view should indicate the need for TV repair $^{6}$ and that TV repair should be accomplished in patients with preoperative atrial fibrillation (AF).

Management guidelines indicate a move toward more aggressive treatment of TR. ${ }^{7}$ For patients undergoing left-side valve surgery, TV repair or replacement is universally recommended in the presence of severe TR or tricuspid annular dilatation because it does not resolve spontaneously after correction of MV disease as once believed. ${ }^{1,8,9}$ In those 


$$
\begin{aligned}
& \text { Abbreviations and Acronyms } \\
& \begin{aligned}
\mathrm{AF} & =\text { atrial fibrillation } \\
\mathrm{ASD} & =\text { atrial septal defect } \\
\mathrm{MV} & =\text { mitral valve } \\
\mathrm{NYHA} & =\text { New York Heart Association } \\
\mathrm{TR} & =\text { tricuspid regurgitation } \\
\mathrm{TV} & =\text { tricuspid valve }
\end{aligned}
\end{aligned}
$$

with isolated severe TR, surgery is recommended in the presence of symptoms or progressive right ventricular dilatation or dysfunction. ${ }^{2,3,5,7,8,10-12}$

Because reoperation for recurrent isolated TR carries high mortality rates (up to $37 \%$ ), TV surgery is not routinely offered to many patients. ${ }^{1,13}$ As minimally invasive approaches for treatment of aortic and MV disease are developing, parallel alternative approaches for TR may be necessary, especially for those patients with high surgical risk.

Since 2005 we have performed TV operations, isolated or in association with other procedures, through a right minithoracotomy using the heart-port platform as the procedure of choice. In this report, early and long-term results of this approach in high-risk patients are examined.

\section{MATERIALS AND METHODS}

Between November 2005 and December 2011 at the University of Turin, 64 patients (19 male, 45 female; mean age, $63.2 \pm 12.8$ years) underwent TV surgery through a lateral right mini-thoracotomy using a nonsternotomy minimally invasive technique. During the same study period, 294 patients underwent TV procedures (combined or isolated) through a standard median sternotomy. The number of TV surgeries in median sternotomy progressively and significantly decreased. For the past 2 years, all isolated TV operations have been performed with a minimally invasive approach, and combined procedures with the same technique significantly increased in the study period (data not shown). Data from all patients were retrospectively reviewed. Preoperative clinical and echocardiographic characteristics are listed in Table 1. Mean preoperative additive European System for Cardiac Operative Risk Evaluation was $7.3 \pm 2.9$, and logistic European System for Cardiac Operative Risk Evaluation was $11.6 \% \pm 11.7 \%$. Predicted mortality of patients undergoing isolated TV surgeries (16 patients) and patients undergoing concomitant mitral procedures (48 patients) was $9.01 \% \pm 2.25 \%$ and $12.52 \% \pm 13.02 \%$, respectively $(P<.05)$. Mean New York Heart Association (NYHA) functional class was $2.8 \pm 0.9$ (median, 3 ; range, $1-4$ ). More than $50 \%$ of patients (37/64) were in NYHA class III or IV. AF was present in 43 patients $(67.2 \%)$. Mean preoperative degree of TR was $3.3 \pm 1.1$ (median, 4; range, $3-4)$. Mean left ventricular ejection fraction was $58.7 \% \pm 3.5 \%$. Pulmonary hypertension (defined as systolic pulmonary artery pressure $\geq 60 \mathrm{~mm} \mathrm{Hg}$ ) was measured in 27 patients $(42.2 \%)$.

TR severity was based on the absolute size of the regurgitant jet by color flow imaging, the relative size of the regurgitant jet (relative to the right atrium area), and the hepatic venous flow pattern, as recommended by the guidelines of the American Society of Echocardiography. ${ }^{14,15}$ In addition, tricuspid annulus diameter was measured in the 4-chamber view to confirm the need for TV repair. In our series, mean TV annulus diameter, measured by echocardiography, was $45.5 \pm 7.2 \mathrm{~mm}$.
$\mathrm{TV}$ regurgitation cause was functional in 36 patients $(56.3 \%)$, due to endocarditis in 2 patients $(3.1 \%)$, and due to rheumatic disease in 24 patients $(37.5 \%)$. Two patients $(3.1 \%)$ showed prosthesis dysfunction.

Forty patients $(62.5 \%)$ underwent reoperations, $8(20.0 \%)$ of whom had undergone 2 previous cardiac surgeries and 12 (30.0\%) of whom had undergone 3 or more previous operations; all were valve surgeries, with no previous CABG. In 12 patients $(30.0 \%)$, it was a previous TV procedure: $2 \mathrm{TV}$ replacements, $1 \mathrm{TV}$ exeresis for endocarditis, and $9 \mathrm{TV}$ repairs (2 Kay procedures and 7 De Vega procedures).

Isolated TV regurgitation was present in 16 patients $(25.0 \%)$. One of these patients had prosthesis valve deterioration. Concomitant cardiac diseases were diagnosed in the remaining 48 patients $(75.0 \%)$. A total of 42 patients $(65.6 \%)$ had concomitant severe mitral regurgitation (native MV regurgitations in 28 , prosthesis valve deterioration in 9 , and mechanical prosthesis dysfunction in 5). Five patients $(7.8 \%)$ had a concomitant patent foramen ovale or an atrial septal defect (ASD), and 1 patient had a left atrial myxoma $(1.5 \%)$.

All patients were screened preoperatively for adequate vascular access by an additional aorto-iliac and femoral artery angiography at the time of cardiac catheterization or by angio-computed tomography scan. Arterial cannulation was obtained through the femoral artery with an Endo-return cannula (EndoReturn arterial cannula 21F or 23F; Edwards Lifesciences, Irvine, Calif) in 43 patients $(67.2 \%)$ and a standard femoral cannula in 16 patients $(15.6 \%)$. In 5 patients $(7.8 \%)$, direct transthoracic aortic cannulation was obtained with the EndoDirect cannula (EndoDirect arterial cannula 24F; Edwards Lifesciences). In case of cannulation with the EndoReturn and EndoDirect systems, aortic clamping was obtained with endoaortic balloons (EndoClamp aortic catheter, Edwards Lifesciences). In case of standard femoral artery cannulation, a Chitwood transthoracic clamp (Scanlan International, St. Paul, Minn) was used. The decision on the type of clamping to use (EndoReturn clamp, EndoDirect clamp, Chitwood clamp) was based on the anatomy of the sinotubular junction, the aorto-iliac-femoral anatomy, the chest structure, and history of cardiothoracic procedures. For example, in case of tortuous and atheromatous aorto-iliac-femoral anatomy, an endovascular clamping technique with the EndoDirect kit was preferred; in case of previous cardiac procedures with a normal vascular anatomy, a peripheral endovascular approach (EndoReturn) was predominantly; in case of a dilated ascending aorta (diameter $>38-40 \mathrm{~mm}$ ), a Chitwood transthoracic clamp was predominantly used. The selection of clamps was patient oriented and independent of the learning curve.

Venous return was routinely obtained with a double cannulation (jugular and femoral). Jugular cannulation was always achieved percutaneously using a $17 \mathrm{~F}$ or $21 \mathrm{~F}$ Medtronic (Minneapolis, Minn) cannula or a $18 \mathrm{~F}$ or 20F Edwards Lifesciences cannula (OptiSite). Femoral cannulation was performed percutaneously in all cases of simultaneous EndoDirect aortic cannulation. In case of EndoReturn or standard femoral artery cannulation, a minimal $(3 \mathrm{~cm})$ groin incision was necessary, and both arterial and femoral cannulations were directly performed using the Seldinger technique through two 4/0 Prolene purse strings. Femoral vein cannulation was obtained with QuickDraw venous cannulas (Edwards Lifesciences). The technique of direct aortic cannulation with the EndoDirect system has been described by Glower and colleagues. ${ }^{16,17}$ The port access surgical technique used at the University of Turin has been described. ${ }^{18}$ Before opening the right atrium, the superior and inferior venae cavae were occluded. Snaring was obtained by placing tourniquets around the superior and inferior venae cavae in 56 patients $(87.5 \%)$ or placing endovascular balloons (7F, $65 \mathrm{~cm}$; Boston Scientific, Natick, Mass) in 8 patients $(12.5 \%)$. The endovascular balloons are delivered under transesophageal echocardiography into the right atrium from the right femoral vein (through a $7 \mathrm{~F}$ introducer) and from the right jugular vein (through a Y-modified cannula). This last approach was preferred in case of previous cardiac surgeries to avoid dangerous dissections. In case of concomitant operation (MV or myxoma), the left atrium was accessed first. After completing the leftsided operation, the left atrium was closed and complete de-airing was 
TABLE 1. Preoperative clinical and echocardiographic characteristics $(N=64$ patients $)$

\begin{tabular}{lc}
\hline Age, y (mean, SD) & $63.2 \pm 12.8$ \\
Female sex (n, \%) & $45(70.3 \%)$ \\
Diabetes (n, \%) & $12(18.7 \%)$ \\
Renal failure (n, \%) & $9(14.1 \%)$ \\
Hypertension (n, \%) & $42(65.6 \%)$ \\
COPD (n, \%) & $3(4.7 \%)$ \\
Pulmonary hypertension ( $\geq 60$ mm Hg) (n, \%) & $27(42.2 \%)$ \\
AF (n, \%) & $43(67.2 \%)$ \\
Cumulative additive euroSCORE (mean, SD) & $7.3 \pm 2.9$ \\
Cumulative log euroSCORE (mean, SD) & $11.6 \pm 11.7$ \\
NYHA class (mean, SD) & $2.8 \pm 0.9$ \\
class I/II (n, \%) & $27(42.2 \%)$ \\
class III/IV (n, \%) & $37(57.8 \%)$ \\
TV grade (mean, SD) & $3.3 \pm 1.1$ \\
TV annulus (mm) (mean, SD) & $45.5 \pm 7.2$ \\
Ejection fraction (mean, SD) & $58.7 \pm 3.5$ \\
Ejection fraction $<50 \%$ & $7(10.9 \%)$ \\
Native MV disease & $28 / 64(43.7 \%)$ \\
MV stenosis & $10 / 28(35.7 \%)$ \\
MV regurgitation & $18 / 28(64.3 \%)$ \\
Redo (n, \%) & $40(62.5 \%)$ \\
1st redo (n, \%) & $20 / 40(50 \%)$ \\
2nd redo (n, \%) & $8 / 40(20 \%)$ \\
3rd or more redo (n, \%) & $12 / 40(30 \%)$ \\
\hline SD, Standard deviation; COPD, chronic obstructive pulmonary disease; AF, atrial \\
fibrillation; euroSCORE, European System for Cardiac Operative Risk Evaluation; \\
NYHA, New York Heart Association; $T V$, tricuspid valve; $M V$, mitral valve.
\end{tabular}

obtained. After declamping the aorta, the tricuspid operation was performed on the beating heart in 33 patients $(51.5 \%)$ ( 16 isolated TV surgeries, 17 concomitant TV surgeries).

After hospital discharge, all patients underwent annual follow-up. Postoperative NYHA class was evaluated, and echocardiographic examination was performed in all cases. This retrospective study was approved by our institutional ethics committee (institutional review board).

\section{Statistical Analysis}

Descriptive statistics for categoric variables are reported as frequency and percentage, and continuous variables are given as mean and standard deviation. Survival was calculated using the Kaplan-Meier method. Comparison between patients who were treated for isolated TV disease and those who had concomitant procedures and between TV repair and replacement were made using the log-rank test.

\section{RESULTS}

$\mathrm{TV}$ repair was performed in 35 patients $(54.7 \%)$. In 33 of 35 cases, a prosthetic ring was used (mean ring size, $30.7 \pm 2.0$; range, 28-34), whereas in the remaining 2 patients a De Vega annuloplasty was carried out. Three of 35 were TV re-repairs with prosthetic ring positioning ( 2 previous De Vega procedures and 1 Kay procedure). TV replacement with bioprosthesis was performed in $27 \mathrm{pa}-$ tients $(42.2 \%)$, whereas the remaining 2 patients $(3.1 \%)$ underwent bioprosthetic replacement (mean prosthetic size, $29.1 \pm 2.5$; range, 25-33). Concomitant procedures (48/64 cases) included MV surgery (14 MV repairs, 14 MV
TABLE 2. Type of surgical procedures and operative data (n, \%) ( $N=64$ patients)

\begin{tabular}{lc}
\hline TV repair & $35(54.7 \%)$ \\
Annular ring & $33 / 35(94.3 \%)$ \\
De Vega annuloplasty & $2 / 35(5.7 \%)$ \\
TV replacement & $27(42.2 \%)$ \\
Tricuspid prosthesis replacement & $2(3.1 \%)$ \\
Isolated TV procedures & $16(25 \%)$ \\
Combined procedures & $48(75 \%)$ \\
MV repair & $14(29.2 \%)$ \\
MV replacement & $14(29.2 \%)$ \\
Mitral prosthesis replacement & $14(29.2 \%)$ \\
ASD closure & $5(10.4 \%)$ \\
Myxoma resection & $1(2 \%)$ \\
TV procedures on beating heart & $33(51.5 \%)$ \\
Isolated TV procedures on beating heart & $16 / 16(100 \%)$ \\
Combined procedures on beating heart & $17 / 48(35.4 \%)$ \\
AF cryoablation & $5 / 43$ preoperative \\
Conversion to sternotomy & AF $(11.6 \%)$ \\
$T V$, Tricuspid valve; $M V$, mitral valve; $A S D$, atrial septal defect; $A F$, atrial fibrillation.
\end{tabular}

replacements, and 14 mitral prosthesis replacements), ASD closure (5 patients), and myxoma exeresis (1 patient). Surgical cryoablation of AF was performed in only 5 patients $(5 / 43,11.6 \%)$. The remaining 38 patients $(88.4 \%)$ were not treated for AF because of a left atrium diameter grater than $60 \mathrm{~mm}$ and a long-standing persistent $\mathrm{AF}$.

At the end of the procedure, intraoperative transesophageal echocardiography showed no or trivial TV regurgitation in all patients who underwent TV repair (35 patients). No leaks were observed in case of TV (27 patients) or prosthetic replacement (2 patients).

Mean duration of cardiopulmonary bypass was $135.4 \pm 41.9$ minutes. In patients undergoing operation for concomitant procedures, the mean crossclamp time was $87.4 \pm 27.4$ minutes (48/64 patients: $75 \%$ ); in such cases, tricuspid surgery was then performed on beating heart in 17 patients $(17 / 48[36.2 \%])$. All the isolated TV procedures (16 patients) were carried out on pump and on beating heart.

Conversion to sternotomy occurred as the result of strong right chest adhesions in 1 patient $(1.6 \%)$ who had undergone previous cardiac surgery. No other complications occurred during surgery. Operative characteristics are listed in Table 2.

Perioperative morbidity was relatively low (Table 3). A major neurologic event (stroke) occurred in 1 patient $(1.6 \%)$ who underwent standard femoral artery cannulation with a transthoracic aortic clamp. Five patients underwent reoperation for bleeding $(7.8 \%)$. Hemodialysis for acute renal failure was necessary in 5 patients $(7.8 \%)$. One patient $(1.6 \%)$ required pacemaker positioning for atrioventricular block. All 5 patients who underwent AF cryoablation were in sinus rhythm at discharge. New postoperative onset of $\mathrm{AF}$ 
TABLE 3. Postoperative outcomes $(N=64$ patients)

\begin{tabular}{lc}
\hline Hospital mortality (n, \%) & $5(7.8 \%)$ \\
Length of postoperative stay (d) (mean, SD, median) & $14.1 \pm 19.0(8)$ \\
Reoperation for bleeding (n, \%) & $5(7.8 \%)$ \\
Stroke (n, \%) & $1(1.6 \%)$ \\
Acute renal failure (n, \%) & $5(7.8 \%)$ \\
Blood loss (mL) & $471 \pm 382$ \\
Pacemaker requirement (n, \%) & $1(1.6 \%)$ \\
\hline
\end{tabular}

$S D$, Standard deviation.

occurred in 11 patients $(17.2 \%)$ with conversion to sinus rhythm after intravenous amiodarone administration in all cases.

Median intensive care unit stay was 1.0 day, and median postoperative hospital stay was 8 days. Mean drain blood loss was $471.5 \pm 382.5 \mathrm{~mL}$. Overall hospital mortality, defined as death at any time before discharge from the hospital or within 30 days of operation, was $7.8 \%$ (5/64 patients) (Table 3 ). The causes of death were mainly related to severe heart failure and poor cardiac output with consequent multiorgan failure ( 3 cases); 1 patient died of sepsis, and 1 patient died after a major neurologic event. Patients who underwent TV repair had a hospital mortality of $2.8 \%$ (1/35 patients), and patients who underwent TV replacement had a hospital mortality of $13.8 \%$ (4/29 patients). Patients who underwent isolated TV procedures had no hospital mortality, and patients who underwent associated TV procedures had a hospital mortality of $10.4 \%$ (5/48 patients). All patients discharged were followed up for $21.5 \pm 36.9$ months.

Early and late postoperative echocardiographic analyses showed trivial or no regurgitation in 62 patients $(96.9 \%)$; only 2 patients with their TV repaired had mild TR (data not shown). Mean NYHA functional class at follow-up was $1.4 \pm 0.7$.

Freedom from reoperation for TV disease was $100 \%$. Kaplan-Meier analysis revealed an overall 5-year survival of $81.3 \%$ and a 5-year freedom from reoperation of $100 \%$. Survival at 5 years was $76.6 \%$ for patients undergoing combined surgery versus $94.1 \%$ for patients undergoing tricuspid surgery alone $(P=.17)$ (Figure 1$)$.

The comparison between patients who underwent isolated TV surgeries and patients who underwent combined procedures in regard to preoperative patient characteristics, echocardiographic measurements, and intraoperative and postoperative findings revealed no statistical difference except for predictive mortality, which was $9.01 \% \pm 2.25 \%$ and $12.52 \% \pm 13.02 \%$, respectively $(P<.05)$ (data not shown).

\section{DISCUSSION}

The tricuspid valve has been commonly referred to as the forgotten valve in comparison with the left-sided valves. Nevertheless, the TV is gaining more attention. Cardiac operation through a right mini-thoracotomy has been used

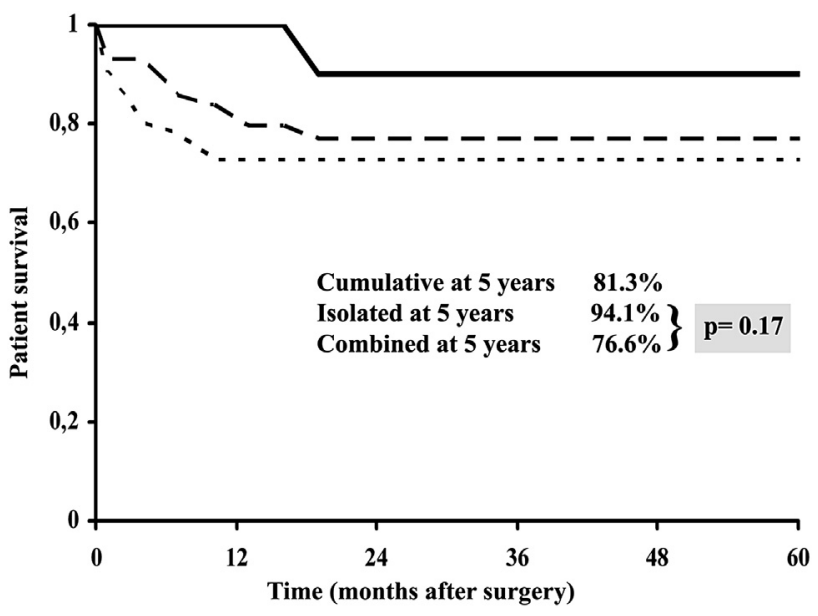

FIGURE 1. Cumulative Kaplan-Meier estimate survival (dashed line). Kaplan-Meier survival for patients who underwent isolated TV surgery (continuous line) and combined procedures (dotted line).

increasingly by many centers in the world. Excellent midterm results have been published for MV operations using this technique. ${ }^{19-21}$ Most of these studies reported less wound infection, less bleeding, less pain, and a more expeditious return to normal life. However, data are lacking on TV operations using a minimally invasive approach. Cardiologists and cardiac surgeons increasingly recognize it has an important entity in structural heart disease. ${ }^{22}$

We present our results on a series of minimally invasive TV operations during a 5-year period. We have considered both TV operations and tricuspid operations associated with other cardiac procedures, such as MV repair/replacement, ASD closure, and myxoma removal. The aim of our study is to demonstrate the feasibility, effectiveness, and safety of such interventions using the port access platform in patients at high surgical risk. Furthermore, a brief comparison between isolated versus combined tricuspid surgeries was done and revealed no differences in terms of preoperative, intraoperative, and postoperative characteristics. The only statistically significant variable was the predicted mortality, which was higher in the combined group versus isolated tricuspid procedures. This difference did not affect the intraoperative and postoperative results.

The percentage of TV repair is low in our series ( 35 over a total of 64 cases). This is in part due to the patients who had undergone previous TV replacement (2 patients) and those who had rheumatic disease (24 patients) and endocarditis (2 patients). By keeping this in mind, the percentage of patients receiving TV repair is approximately $100 \%(35 / 36$ : $97.2 \%$ ), far more than previously reported by other authors for isolated TV surgeries. ${ }^{4,22-24}$ All 36 had functional TRs. TV repair was performed even in those patients who underwent previous TV repair. This result confirms that a TV repair was performed when possible and that it is feasible with a minimally invasive approach. A right 
mini-thoracotomy allows extraordinary exposure of the TV and its apparatus, ensuring the performance of any surgical procedure and thus increasing the repair rate.

In this study, few patients were treated for AF. An MV procedure was associated in all cases. The decision not to treat AF was made in those patients with an extremely enlarged left atrium $(>60 \mathrm{~mm}$ or $>100 \mathrm{~mL})$ and in those who had undergone previous cardiac procedures. In this case, the port-access technique allows the TV to be operated with limited dissection of adhesions. AF treatment requires more extensive isolation of cardiac structures, and this can be extremely dangerous in up to 3 re-do operations.

Although Lee and colleagues ${ }^{22}$ demonstrated different findings, in our experience, the cardiopulmonary bypass and aortic clamp times are acceptable and comparable to the operative results reported by other authors in case of operation performed through a median sternotomy ${ }^{23}$ or a minimally invasive approach for isolated TV procedures. ${ }^{4}$ These data are encouraging considering that additional cardiac procedures were associated with TV surgery and that in most of the cases $(62.4 \%)$ a previous cardiac procedure was done. ${ }^{18}$

We found a low incidence of postoperative adverse events compared with other reported series. ${ }^{22}$ Major neurologic complications occurred in only 1 case in which a femoral cannulation was used. The event could have been a consequence of peripheral cannulation and retrograde perfusion. Peripheral cannulation was associated with a higher incidence of stroke in some early reports of valve surgery through a right mini-thoracotomy. ${ }^{18,25,26}$ The rate of reoperation for bleeding still remains slightly high $(7.5 \%)$ but in line with other series. ${ }^{22}$ All patients who underwent reoperation for bleeding were redo cases. This could be explained by the fact that more than $60 \%$ were redo cases (up to 3 redo cases in $30 \%$ of the patients) with the need for dissection of strong adhesions. In the first phase of our experience, caval snaring was obtained by putting tapes around both venae cavae with the need of extended dissections. Since 2008, we have adopted a new technique of bicaval snaring using endovascular balloon catheters (Equalizer Occlusion Balloon Catheter; Boston Scientific) that ensure endocaval occlusion with no need for extensive dissections. We have adopted this system in redo cases with success. The endocaval balloon does not interfere in the surgical field, and no balloon dislocation has been observed. The introduction in our common practice of endocaval occluders in redo cases has allowed a significant reduction in adhesion dissection with minimal blood loss (before 2008, reoperation for bleeding occurred in 4 cases, whereas only 1 case occurred after 2008). Moreover, it avoids air entrapment in the venous line and the risk of arterial microembolism events, which are more common in case of right atrium opening using vacuum assist without caval snaring.

In our series, we report a cumulative 30-day mortality rate of $7.8 \%$. This is in line with other published data with mortality rates ranging from $4 \%$ to $22 \%{ }^{4,14,23,26,27}$ Lee and colleauges ${ }^{22}$ report a low mortality rate at 30 days $(2.1 \%)$ combining repair and replacement. Our results show a mortality rate of $2.8 \%$ and $13.8 \%$ for TV repair and replacement, respectively. In TV replacement, predictive mortality is $14.12 \% \pm 13.23 \%$. Other authors have reported series with higher mortality rates with TV replacement. ${ }^{28,29}$ The mortality rate in our study is $0 \%$ for isolated TV surgeries and $10.4 \%$ for combined procedures. Rankin and colleagues $^{30}$ report from the Society of Thoracic Surgeons database a 30-day mortality rate of $10.7 \%$ for isolated TV surgeries versus $10.8 \%$ for combined MV procedures.

Unlike other reports, ${ }^{22,28,31}$ the overall survival at 5 years in our series is higher: ranging from $81.3 \%$ to $94.1 \%$ for patients undergoing TV surgery alone and $76.6 \%$ for patients undergoing combined surgery $(P=.17)$. No statistical difference was observed in terms of late survival for isolated TV surgeries versus combined procedures. In addition, overall freedom from reoperation was $100 \%$ at 5 years. Complete echocardiographic and clinical follow-up confirmed optimal results with a significant improvement in functional class and no residual regurgitation in more than $95 \%$ of the cases. Early and late outcomes suggest that the minimally invasive approach to tricuspid surgery is safe and effective.

\section{Study Limitations}

This is a single-center retrospective study, and a small number of patients were treated. A longer follow-up is needed to confirm long-term results and TV repair durability. Other limitations to the use of this surgical approach are mainly related to a prolonged learning curve and the cost of the devices. Embolism of air remains a concern when left cardiac cavities are opened. Careful de-airing can reduce this risk. Moreover, the operating field can be continuously flooded with carbon dioxide using a special trocar that allows the insertion of both a venting cannula and a carbon dioxide line.

\section{CONCLUSIONS}

In this series of high-risk patients undergoing TV operations, the minimally invasive approach is safe, feasible, and effective; ensures low perioperative morbidity and mortality; and ensures low rates of recurrent TR and late mortality. The nonsternotomy minimally invasive technique has become the standard of care at the University of Turin for isolated or combined TV procedures, especially in case of redo procedures.

We would like to thank Prof. Laura Bergamasco for statistical analysis and writing assistance.

\section{References}

1. Yilmaz O, Suri RM, Dearani JA, Sundt TM III, Daly RC, Burkhart HM, et al. Functional tricuspid regurgitation at the time of mitral valve repair for 
degenerative leaflet prolapse: the case for a selective approach. $J$ Thorac Cardiovasc Surg. 2011;142:608-13.

2. Irwin RB, Luckie M, Khattar RS. Tricuspid regurgitation: contemporary management of a neglected valvular lesion. Postgrad Med J. 2010;86: 648-55.

3. Desai RR, Vargas Abello LM, Klein AL, Marwick TH, Krasuski RA, Ye Y, et al. Tricuspid regurgitation and right ventricular function after mitral valve surgery with or without concomitant tricuspid valve procedure. J Thorac Cardiovasc Surg. 2012 Sep 22. [Epub ahead of print].

4. Seeburger J, Borger MA, Passage J, Misfeld M, Holzhey D, Noack T, et al. Minimally invasive isolated tricuspid valve surgery. J Heart Valve Dis. 2010;19: 189-93.

5. Vargas Abello LM, Klein AL, Marwick TH, Nowicki ER, Rajeswaran J, Puwanant $\mathrm{S}$, et al. Understanding right ventricular dysfunction and functional tricuspid regurgitation accompanying mitral valve disease. J Thorac Cardiovasc Surg. 2012 May 22. [Epub ahead of print].

6. Dreyfus GD, Chan KM. Functional tricuspid regurgitation: a more complex entity than it appears. Heart. 2009;95:868-9.

7. Bonow RO, Carabello BA, Kanu C, de Leon AC Jr, Faxon DP, Freed MD, et al. ACC/AHA 2006 guidelines for the management of patients with valvular heart disease: a report of the American College of Cardiology/American Heart Association Task Force on Practice Guidelines (writing committee to revise the 1998 Guidelines for the Management of Patients With Valvular Heart Disease): developed in collaboration with the Society of Cardiovascular Anesthesiologists: endorsed by the Society for Cardiovascular Angiography and Interventions and the Society of Thoracic Surgeons. Circulation. 2006; 114:e84-231.

8. Dreyfus GD, Corbi PJ, Chan KM, Bahrami T. Secondary tricuspid regurgitation or dilatation: which should be the criteria for surgical repair? Ann Thorac Surg. 2005;79:127-32.

9. Ro SK, Kim JB, Jung SH, Choo SJ, Chung CH, Lee JW. Mild-to-moderate functional tricuspid regurgitation in patients undergoing mitral valve surgery. J Thorac Cardiovasc Surg. 2012 Aug 31. [Epub ahead of print].

10. Raja SG, Dreyfus GD. Basis for intervention on functional tricuspid regurgitation. Semin Thorac Cardiovasc Surg. 2010;22:79-83.

11. Rogers JH, Bolling SF. The tricuspid valve: current perspective and evolving management of tricuspid regurgitation. Circulation. 2009;119:2718-25.

12. Yurekli I, Kestelli M, Gunes T, Akyuz M. Some issues about the decisionmaking process for tricuspid valve repair. J Thorac Cardiovasc Surg. 2012; 144:287.

13. Pfannmuller B, Moz M, Misfeld M, Borger MA, Funkat AK, Garbade J, et al. Isolated tricuspid valve surgery in patients with previous cardiac surgery. J Thorac Cardiovasc Surg. 2012 Aug 28. [Epub ahead of print].

14. Bernal JM, Gutierrez-Morlote J, Llorca J, San Jose JM, Morales D, Revuelta JM. Tricuspid valve repair: an old disease, a modern experience. Ann Thorac Surg. 2004;78:2069-75.

15. Kim JB, Jung SH, Choo SJ, Chung CH, Lee JW. Clinical and echocardiographic outcomes after surgery for severe isolated tricuspid regurgitation. J Thorac Cardiovasc Surg. 2012 Aug 4. [Epub ahead of print].
16. Glower DD, Clements FM, Debruijn NP, Stafford-Smith M, Davis RD Landolfo KP, et al. Comparison of direct aortic and femoral cannulation for port-access cardiac operations. Ann Thorac Surg. 1999;68:1529-31.

17. Glower DD, Komtebedde J, Clements FM, Debruijn NP, Stafford-Smith M, Newman MF. Direct aortic cannulation for port-access mitral or coronary artery bypass grafting. Ann Thorac Surg. 1999;68:1878-80.

18. Ricci D, Pellegrini C, Aiello M, Alloni A, Cattadori B, D’Armini AM, et al. Portaccess surgery as elective approach for mitral valve operation in re-do procedures. Eur J Cardiothorac Surg. 2010;37:920-5.

19. Grossi EA, Galloway AC, LaPietra A, Ribakove GH, Ursomanno P, Delianides J, et al. Minimally invasive mitral valve surgery: a 6-year experience with 714 patients. Ann Thorac Surg. 2002;74:660-4.

20. Modi P, Hassan A, Chitwood WR Jr. Minimally invasive mitral valve surgery: a systematic review and meta-analysis. Eur J Cardiothorac Surg. 2008;34: 943-52.

21. Seeburger J, Borger MA, Falk V, Kuntze T, Czesla M, Walther T, et al. Minimal invasive mitral valve repair for mitral regurgitation: results of 1339 consecutive patients. Eur J Cardiothorac Surg. 2008;34:760-5.

22. Lee TC, Desai B, Glower DD. Results of 141 consecutive minimally invasive tricuspid valve operations: an 11-year experience. Ann Thorac Surg. 2009;88: 1845-50.

23. Guenther T, Noebauer C, Mazzitelli D, Busch R, Tassani-Prell P, Lange R. Tricuspid valve surgery: a thirty-year assessment of early and late outcome. Eur J Cardiothorac Surg. 2008;34:402-9.

24. Staab ME, Nishimura RA, Dearani JA. Isolated tricuspid valve surgery for severe tricuspid regurgitation following prior left heart valve surgery: analysis of outcome in 34 patients. J Heart Valve Dis. 1999;8:567-74.

25. Burfeind WR, Glower DD, Davis RD, Landolfo KP, Lowe JE, Wolfe WG. Mitra surgery after prior cardiac operation: port-access versus sternotomy or thoracotomy. Ann Thorac Surg. 2002;74:S1323-5.

26. Grossi EA, LaPietra A, Ribakove GH, Delianides J, Esposito R, Culliford AT, et al. Minimally invasive versus sternotomy approaches for mitral reconstruction: comparison of intermediate-term results. J Thorac Cardiovasc Surg. 2001;121: 708-13.

27. McCarthy PM, Bhudia SK, Rajeswaran J, Hoercher KJ, Lytle BW, Cosgrove DM, et al. Tricuspid valve repair: durability and risk factors for failure. J Thorac Cardiovasc Surg. 2004;127:674-85.

28. Moraca RJ, Moon MR, Lawton JS, Guthrie TJ, Aubuchon KA, Moazami N, et al. Outcomes of tricuspid valve repair and replacement: a propensity analysis. Ann Thorac Surg. 2009;87:83-9.

29. Rizzoli G, Vendramin I, Nesseris G, Bottio T, Guglielmi C, Schiavon L. Biolog ical or mechanical prostheses in tricuspid position? A meta-analysis of intrainstitutional results. Ann Thorac Surg. 2004;77:1607-14.

30. Rankin JS, Hammill BG, Ferguson TB Jr, Glower DD, O’Brien SM, DeLong ER, et al. Determinants of operative mortality in valvular heart surgery. $J$ Thorac Cardiovasc Surg. 2006;131:547-57.

31. Filsoufi F, Anyanwu AC, Salzberg SP, Frankel T, Cohn LH, Adams DH. Longterm outcomes of tricuspid valve replacement in the current era. Ann Thorac Surg. 2005;80:845-50. 